\title{
ANALYSIS OF MECHANICAL STRUCTURES USING PLATE FINITE ELEMENT METHOD UNDER DIFFERENT BOUNDARY CONDITIONS
}

\author{
Fareh HAMRIT ${ }^{1}$, Brahim NECIB ${ }^{1,2}$ \\ ${ }^{1}$ Laboratory of Mechanics, University of Constantine 1, Algeria, \\ ${ }^{2}$ Mechanical Engineering Department, Faculty of Technology Sciences, University of Constantine 1, Algeria \\ necibbrahim2004@yahoo.fr
}

\begin{abstract}
The mechanical discrete or continuum structures are actually of great importance in the application field of contemporary modern industry. However, during their life time these structures are often subjected to considerable external stresses or to high amplitudes of vibrations which can cause them large deformations and internal stresses which can cause them internal cracking or even their total destruction. In order to avoid these types of problems, the concept of static and dynamic analysis of these structures is recommended, and due to the complexity of their shape and size, the finite element method is the most used. The latter is currently recognized as a very powerful technique for the static and dynamic analysis of discrete or continuous structures of complicated form applied in the field of mechanics, aeronautics, civil engineering, maritime or robotics. Consequently, the calculation and dimensioning of these mechanical systems by the finite element method plays an important role at the service of the industry for possible sizing and prediction of their lifetime. Our work consists of static and dynamic analysis of two-dimensional discrete and continuous mechanical systems using the finite element method based on the main elements of bars, beams and plates, under the effect of external excitations with different boundary conditions. The discrete structures considered are two-dimensional in metallic framework interconnected to the nodes by welding, riveting or bolted under various boundary conditions. Their elements are modeled comparatively by bar elements and beam elements, while for continuous structures the elements are rectangular thin plates with different boundary conditions. The excitation forces are based on periodic, random or impulsive forces and a numerical solution by development of a program to describe the behavior of these structures is realized. The mass and stiffness matrices of all the structures are determined respectively by assembling the bars, beam and plate elements based on the kinetic and deformation energy for each element. The displacements, the node reactions and the axial forces in all the elements as well as the transverse stresses and the eigenvalues of the structures under different boundary conditions were also calculated and good results were obtained compared to those obtained using other software already existing. In fact, analysis using the finite element method will allow the proper dimensioning and design of complex industrial mechanical structures according to different boundary conditions, their internal loading and their vibratory level.
\end{abstract}

Keywords: Structures, vibration modes, finite elements, plate element, assembly, deformation energy, deformation, stress.

\section{INTRUDUCTION}

The continuous structures are composed of plate elements and subjected to external forces or moments. Under the effect of these forces, the structure may be deformed and the internal stresses in each element may occur. These structures are characterized by a finite number of unknown displacements and the forces at the nodes parameters. This method is used also to analyze the three dimensional bodies or cylindrical bodies using plate, triangular or shell elements. In fact, this method is based on the discretization of the structure or continuous body into infinitesimal elements limited by nodes and then by assembling them in order to obtain the overall and the entire structure [1]. Thus, the shape of the structure or the body is obtained in respecting its conditions with the initial limits and applied efforts. In general, the behavior of all the assembled basic members describes all of the behavior of the whole structure or body. The present work consists on the use of this method for static and dynamic analysis of structures under the influence of outside excitation with different boundary conditions. The stiffness and mass matrix of each element is computed, and then assembled to find the overall stiffness and mass matrix of the structure. In fact, the finite element method is known as a very powerful technique used to analysis discrete or continuum structures, in the field of engineering. It is now used in many sectors of the industry, mechanical, civil, aerospace and robotics. This work is devoted to the use of this method for static and dynamic analysis of structures in porches (Plate element) due to excitement outside with different boundary conditions. Understanding this method gives necessity in the development of certain scientific 
knowledge as the theory of elasticity, mechanical environment continues, the strength of materials, structural dynamics, and applied mathematics. If the structure has a complex system of behavior and continues defined by the infinite number of parameters [2], it becomes very difficult to analyze or find the analytical solution. However, the finite element method grows the ability to find the most perfect solution while replacing the continuous system by a discrete system, characterized by a finite number of parameters [3].

In this context, a program of computation based on PASCAL language has been developed. The displacements, forces and reactions at the nodes as well as the axial strengths in each element and the clean fashions of the all structure have been determined under different applied loads and boundary conditions in static and dynamic cases. The obtained results are compared to those founded using existing programs such as "Ansys 17.0" and "Abaqus". A good comparison has been observed.

\section{PROBLEM AND METHODOLOGY}

The systems with one degree of freedom are simply illustrated by the system represented on figure 1 supposed in a vertical plane. $x(t)$ is the displacement of the mass $\mathrm{m}$ starting from the equilibrium position [4].

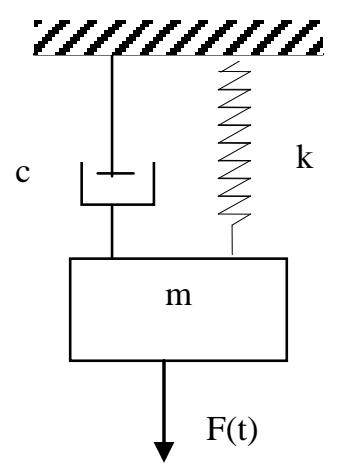

Fig. 1. System with one degree of freedom

The equation of the motion is given by

$$
m \ddot{x}(t)+c \dot{x}(t)+k x(t)=F(t)
$$

The solutions of the characteristic equations are:

$$
r_{1,2}=-\frac{c}{2 m} \pm \frac{\sqrt{c^{2}-4 k m}}{2 m}
$$

Then, we can rewrite the equation of motion without excitation as:

$$
\ddot{x}+2 w_{0} \alpha \dot{x}+w_{0}^{2} x=0
$$

When the system mass-spring without damping is submitted to an excitation, the equation of motion becomes [5]:

$$
m \ddot{x}(t)+k x(t)=F(t)
$$

In our study, the equations of motion of the element plates are formulated in a similar way to equation (3), but with several degrees of freedom, this will be formulated using the matrix form and will be presented as:

$$
[m]\{\ddot{x}\}+[k]\{x\}=\{F(t)\}
$$

Where:

[k]: matrix of rigidity,

[m]: mass matrix ,

$\{\mathrm{x}\}$ : vector of nodal displacements,

$\{\ddot{x}\}$ : nodal acceleration vector,

$\{\mathrm{F}(\mathrm{t})\}$ : nodal applied forces.

\section{MATHEMATICAL FORMULATION}

\subsection{Oscillation forced}

The existence of even low friction, leads irremediably to the extinction of the oscillatory motion by dissipation of energy (internal or external). It is necessary to maintain the movement, to external energy in the form of a so-called power exciter. It will be admitted that (resonator) system does not react on the exciter.

\subsection{Harmonic excitation}

The equation of motion for a damped harmonic oscillator subjected to an external force $\mathrm{F}(\mathrm{t})$.

The simplest case is that when the excitation is a harmonic force in the form:

$$
F(t)=F \cos (w t+\theta)
$$

The general solution of the motion equation is then a linear combination of the general solution of the equation without second member (free oscillations plane), and a particular solution of the equation with second member. As previously, we can rewrite equation (1) as follows:

$$
\ddot{x}(t)+2 \alpha w_{0} \dot{x}(t)+w_{0}{ }^{2} x(t)=\frac{F(t)}{m}
$$

\subsection{Generalized eigen values problem}

Assuming that the discretized system is stationary (coincidence of fixed and linked axes) and by neglecting the depreciation of viscous matrix, the free discrete associated equation of motion will be expressed in the following form [6]:

$$
[m] \ddot{x}(t)+[k] x(t)=0
$$

\section{MODELING OF THE MASSES BY FINITE ELEMENT}

It is possible to arbitrarily concentrate the mass in specific points using the know-how, common sense or intuition, or it is possible to initially give displacement functions. This last idea can be prolonged by adding to it the concept of discretization by finite element. Indeed, it is the displacement field that is interpolated nodal, in an element finite [7]. It is then assumed that the generalized variables or nodal displacement are depending on the time to calculate the kinetic energy at each element. The matrix mass obtained in this way is called matrix of the consistent masses or distributed to the extent. In these conditions, the 
calculation of the kinetic energy is consistent with the strain energy.

\subsection{Application of the finite element method to a rectangular element}

The finite element of the plane state is a rectangular element as shown in figure 2 . The element has a length $a$ and a width $b$ with a constant thickness $h$. Each of the four corners or nodal points has two degrees of freedom $u$ and $v$, witch present the directions of $x$ and $y$ respectively. Thus this element has eight nodal masses (four pairs of $\mathrm{m}_{\mathrm{x}}$ and $\mathrm{m}_{\mathrm{y}}$ ) and eight nodal displacements or nodal degrees of freedom (four pairs of $u$ and $v$ ).
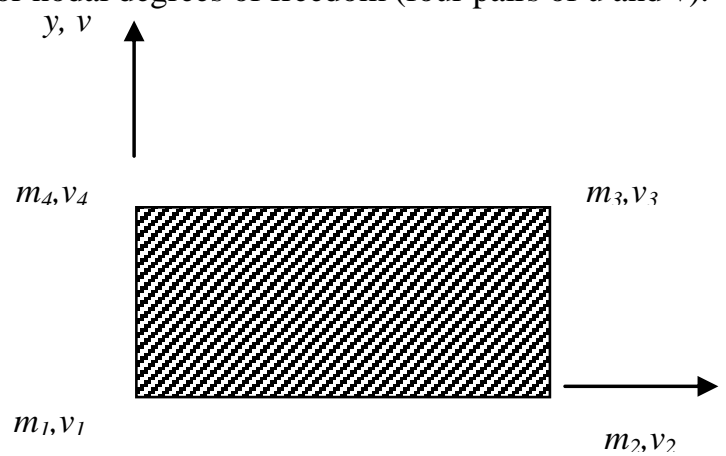

Fig. 2. Rectangular finite element with eight degrees of freedom at a plane state of stress or strain deformation

\subsection{Rigidity matrix of the rectangular element}

The elementary matrix of rigidity can be derived by initially formulating the strain energy deformation $\mathrm{U}$ for the element and then by carrying out the differentiation of the partial equation of this energy with respect to each degree of freedom according to the theorem of Castigliano [13] given by:

$$
F i=\frac{\partial U}{\partial u_{i}}
$$

The strain energy deformation of the finite element is found using a linear displacement at $(\mathrm{x}, \mathrm{y})$ position [8]:

$$
\begin{aligned}
& u(x, y)=c_{1}+c_{2} x+c_{3} y+c_{4} x y \\
& v(x, y)=c_{1}+c_{2} x+c_{3} y+c_{4} x y
\end{aligned}
$$

Where $c_{i}$ are constants found using boundary conditions at each nodal point of the element. The equation (9) can be written as follows:

$$
\begin{aligned}
& u(x, y)=f_{1}(x, y) u+f_{2}(x, y) u_{2}+f_{3}(x, y) u_{3}+f_{4}(x, y) u_{4} \\
& v(x, y)=f_{1}(x, y) v+f_{2}(x, y) v_{2}+f_{3}(x, y) v_{3}+f_{4}(x, y) v_{4}
\end{aligned}
$$

Where the four functions $F(x, y)$ are called the displacement functions obtained as:

$$
\begin{aligned}
& f_{1}(x, y)=\left(1-\frac{x}{a}\right)\left(1-\frac{y}{b}\right) \\
& f_{2}(x, y)=\frac{x}{a}\left(1-\frac{y}{b}\right) \\
& f_{3}(x, y)=\frac{x y}{a b} \\
& f_{4}(x, y)=\left(1-\frac{x}{a}\right) \frac{y}{b}
\end{aligned}
$$

As a result, the tensor strain deformation can be found and expressed as [9]:

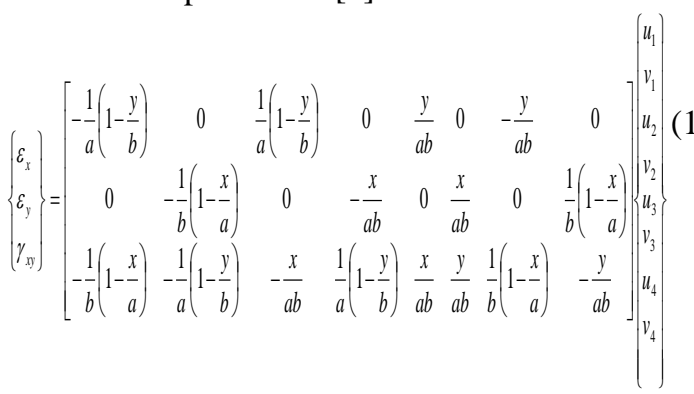

In the matrix form, it can be written follows: $\{\varepsilon\}=[A]\{q\}$

The equations of rigidity for the rectangular element at eight degree of liberty can be found as [10]:

$$
\left\{\begin{array}{l}
\mathrm{F}_{X 1} \\
F_{Y 1} \\
F_{X 2} \\
F_{Y 2} \\
F_{X 3} \\
F_{Y 3} \\
F_{X 4} \\
F_{Y 4}
\end{array}\right\}=\left[\begin{array}{cccccccc}
C_{1} & & & & & & & \\
C_{2} & C_{3} & & & & & & \\
C_{4} & -C_{5} & C_{1} & & & & & \\
C_{5} & C_{6} & -C_{2} & C_{3} & & & & \\
\frac{-C_{1}}{2} & -C_{2} & C_{1} & -C_{5} & C_{1} & & & \\
-C_{2} & \frac{-C_{3}}{2} & C_{5} & C_{8} & C_{2} & C_{3} & & \\
C_{1} & C_{5} & \frac{-C_{1}}{2} & C_{2} & C_{4} & -C_{5} & C_{1} & \\
-C_{5} & C_{8} & C_{2} & \frac{-C_{3}}{2} & C_{5} & C_{6} & -C_{2} & C_{3}
\end{array}\right]\left[\begin{array}{c}
u_{1} \\
v_{1} \\
u_{2} \\
v_{2} \\
u_{3} \\
v_{3} \\
u_{4} \\
v_{4}
\end{array}\right\}(14)
$$

Where:

$$
\begin{aligned}
& C_{1}=\left(\frac{b}{3 a}+\frac{1-v}{6} \frac{a}{b}\right) \frac{E t}{1-v^{2}} \\
& C_{2}=\left(\frac{v}{4}+\frac{1-v}{8}\right) \frac{E t}{1-v^{2}} \\
& C_{3}=\left(\frac{a}{3 b}+\frac{1-v}{6} \frac{b}{a}\right) \frac{E t}{1-v^{2}} \\
& C_{4}=\left(-\frac{b}{3 a}+\frac{1-v}{12} \frac{a}{b}\right) \frac{E t}{1-v^{2}} \\
& C_{5}=\left(\frac{v}{4}-\frac{1-v}{8}\right) \frac{E t}{1-v^{2}} \\
& C_{6}=\left(\frac{a}{6 b}+\frac{1-v}{6} \frac{b}{a}\right) \frac{E t}{1-v^{2}} \\
& C_{7}=\left(\frac{b}{6 a}+\frac{1-v}{6} \frac{a}{b}\right) \frac{E t}{1-v^{2}} \\
& C_{8}=\left(-\frac{a}{3 b}+\frac{1-v}{12} \frac{b}{a}\right) \frac{E t}{1-v^{2}}
\end{aligned}
$$

\section{DETERMINATION OF THE MASS \\ MATRIX}

The determination of the eigen modes of a structure by the finite element technique requires the replacement of the inertial force due to the mass of the structure by the equivalent inertia forces applied to the nodes. 


$$
\{f(x, y, z)\} . f(t)=\left[\Phi(x, y, z)\{\alpha\} \sin \omega_{n} t\right]
$$

The inertia forces per unit volume due to the vibrations of the structure have the following components:

$$
\mathrm{X}=-\rho \frac{\partial^{2} u}{\partial t^{2}}, Y=-\rho \frac{\partial^{2} v}{\partial t^{2}}, Z=-\rho \frac{\partial^{2} \omega}{\partial t^{2}}
$$

Where $\rho$ is the density.

Then, the distributed load vector $\{\mathrm{q}(\mathrm{x}, \mathrm{y}, \mathrm{z})\}$ will be as follows:

$$
\begin{gathered}
\{q(x, y, \omega)\}=\left\{\begin{array}{c}
\rho \frac{\partial^{2} u}{\partial t^{2}} \\
\rho \frac{\partial^{2} v}{\partial t^{2}} \\
\rho \frac{\partial^{2} \omega}{\partial t^{2}}
\end{array}\right\} \\
\left.\{q(x, y, z)\}=-\rho \frac{\partial^{2}}{\partial t^{2}}\left\{\Phi(x, y, z)\{\alpha\} \sin \omega_{n} t\right]\right\} \\
\left.=-\omega_{n}^{2} \rho\left\{\Phi(x, y, z)\{\alpha\} \sin \omega_{n} t\right]\right\}
\end{gathered}
$$

As well as:

$$
\{q(x, y, z)\}=\omega_{n}^{2} \rho\left[\Phi(x, y, z)\left[A_{1}^{-1}\right]\{\delta\} \sin \omega_{n} t\right]
$$

Substituting equation (17) in (18), we obtain:

$$
Q_{c, r}=\{d \delta\}^{T}\left[A_{1}^{-1}\right]^{T} \cdot \int[\Phi(x, y, z)]^{T} \cdot \omega_{n}^{2} \rho[\Phi(x, y, z)]\left[A_{1}^{-1}\right]\{\delta\} \sin \omega_{n} t d v
$$

The virtual work of the equivalent inertial forces applied to the nodes is:

$Q_{c, r}=\{d \delta\}^{T} \cdot\{N\}$

By equalizing the two obtained works, we will have:

$$
\begin{gathered}
\{N\}=\omega_{n}^{2}\left[A_{1}^{-1}\right]^{T}\left[\int_{v}[\Phi(x, y, z)]^{T} \cdot[\Phi(x, y, z)] d v\right] \\
{\left[A_{1}^{-1}\right]\{\delta\} \sin \omega_{n} t} \\
\{N\}=\omega_{n}^{2}\left[M_{e}\right]\{\delta\} \sin \omega_{n} t
\end{gathered}
$$

while:

$$
\left\{M_{e}\right\}=\rho\left[A_{1}^{-1}\right]^{T}\left[\int_{v}[\Phi(x, y, z)]^{T} \cdot[\Phi(x, y, z)] d v\right] \cdot\left[A_{1}^{-1}\right](20)
$$

$\left[M_{e}\right]$ : is the elementary mass matrix for a plate element and can be expressed as follows:

$$
\left[M_{e}\right]=\text { p.a.b.h }\left[\begin{array}{cccccccc}
\frac{1}{9} & \frac{1}{18} & \frac{1}{36} & \frac{1}{18} & 0 & 0 & 0 & 0 \\
\frac{1}{18} & \frac{1}{9} & \frac{1}{18} & \frac{1}{36} & 0 & 0 & 0 & 0 \\
\frac{1}{36} & \frac{1}{18} & \frac{1}{9} & \frac{1}{18} & 0 & 0 & 0 & 0 \\
\frac{1}{18} & \frac{1}{36} & \frac{1}{18} & \frac{1}{9} & 0 & 0 & 0 & 0 \\
0 & 0 & 0 & 0 & \frac{1}{9} & \frac{1}{18} & \frac{1}{36} & \frac{1}{18} \\
0 & 0 & 0 & 0 & \frac{1}{18} & \frac{1}{9} & \frac{1}{18} & \frac{1}{36} \\
0 & 0 & 0 & 0 & \frac{1}{36} & \frac{1}{18} & \frac{1}{9} & \frac{1}{18} \\
0 & 0 & 0 & 0 & \frac{1}{18} & \frac{1}{36} & \frac{1}{18} & \frac{1}{9}
\end{array}\right]
$$

\section{EXEMPLES OF APPLICATIONS}

For the resolution of a plane elasticity problem by the finite element method, we use the Pascal program and the results are compared to our developed program based on the sap2000 software for an isotropic solid plate in a plane state of stresses. An elastic problem is solved once the displacement vector in any point of the solid and the forces at each point of solid are known. In this problem, the boundary conditions are known. Some examples are then considered to examine our developed program.

the considered example presents a homogeneous plate which is discretized in equal rectangular elements, $\mathrm{n}$ elements in the $\mathrm{X}$ axis and $\mathrm{m}$ elements in the $\mathrm{Y}$ axis. For a static case, we can calculate the nodal displacements $\left(u_{i}, v_{i}\right)$, the reactions at each nodal points and the stress in the medium of each element. Then for the dynamic case, we calculate the eigen frequencies and the eigen values. The comparison of the obtained results between our developed program and the SAP2000 are presented. The study of the examples related to a rectangular plate in a plane state of stress under various boundary conditions is developed to analyze the thin plate structure by the finite element method. The discretization of this plate is done in very small rectangular elements such as presented in figure 3 .

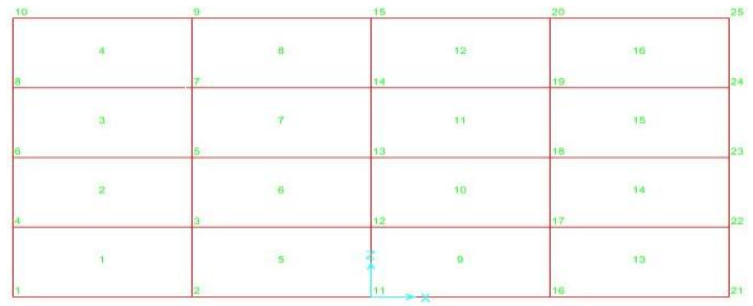

Fig. 3. Discretization of the thin plate (rectangular finite elements)

As presented in figure 5, the considered rectangular plate is recessed at one of its ends and free on the rest of its perimeter. The characteristics of the elastic material are defined by the length of the plate equal to $a=2 \mathrm{~m}$, the width $b=1 \mathrm{~m}$, and thickness: $h=0.05 \mathrm{~m}$. The Young's modulus is equal to $E=2.10^{11} \mathrm{~N} / \mathrm{m}^{2}$, and the density is equal to $\rho=7800 \mathrm{~kg} / \mathrm{m}^{3}$, with a modulus of fish $\mu=0.3$. For the finite element resolution, we have use a regular mesh composed of 16 elements and 25 nodes. For our analysis and for a software validation Abaqus, we have used a mesh defined by 200 elements and 231 nodes. Indeed, we have used a tetrahedral mesh for Ansys 17.0 software on one side to see the effect of the mesh on the results obtained and on the other side or even the differences between the mesh types. And the elements with transverse shear. Its elements use a quadratic approximation of out of plane displacement and rotations. Each element has eight nodes and thus 58 degrees of freedom, with the boundary conditions obtaining the results, nodal displacements, reactions to the supports, axial 
forces in the elements and the Eigen modes of the whole structure.

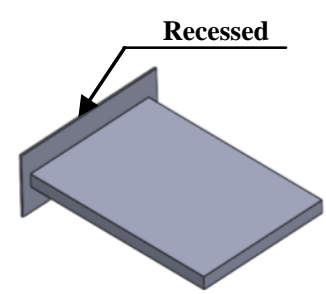

(a) Recessed - free

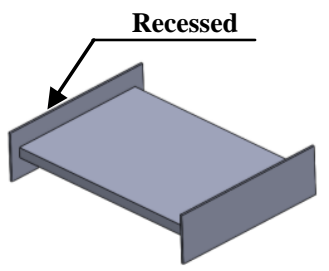

(b) recessed - recessed

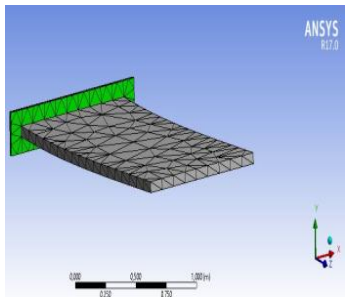

(a) Ansys

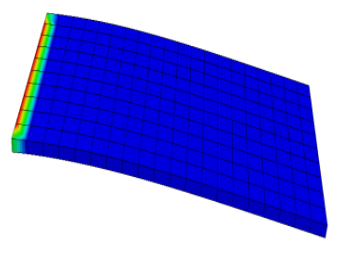

(b) Abaqus.
Fig. 5. First mode of the structure A

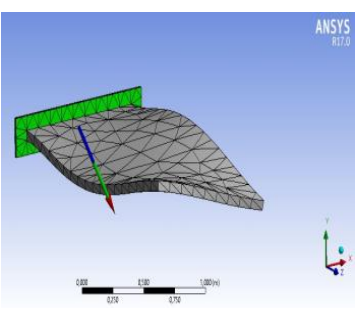

(a) Ansys

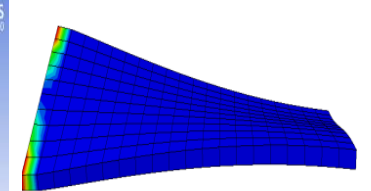

(b) Abaqus
Fig. 6. Second mode of structure A

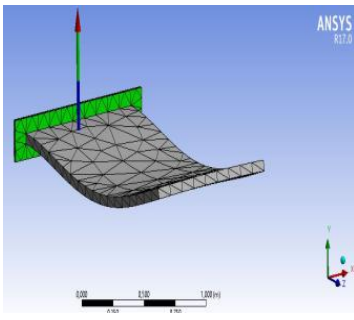

(a) Ansys

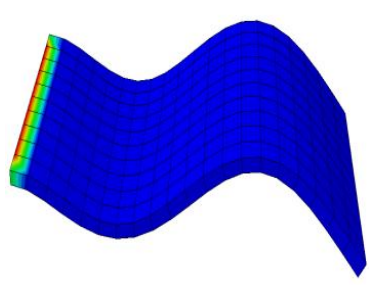

(b) Abaqus
Fig. 7. Third mode of structure A

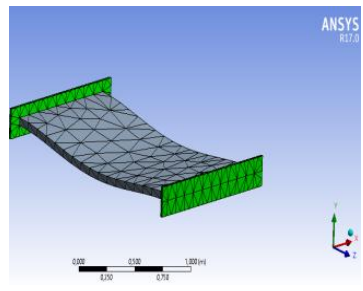

(a) Ansys

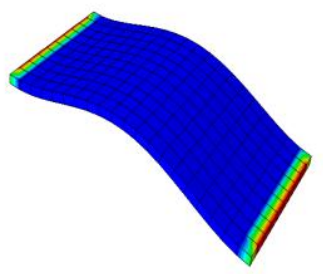

(b) Abaqus

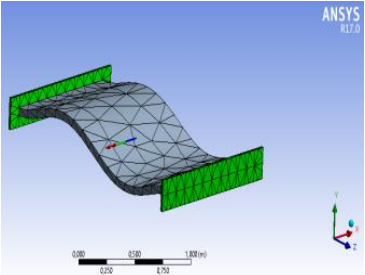

(a) Ansys

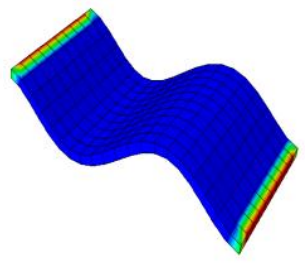

(b) Abaqus
Fig. 9. Second mode of structure B

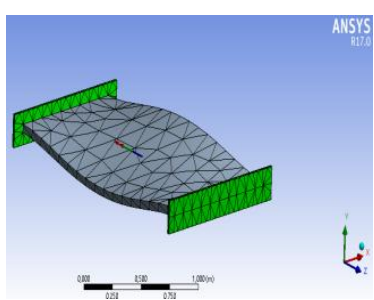

(a) Ansys

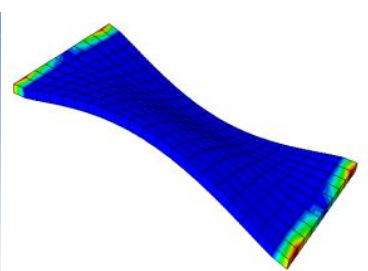

(b) Abaqus
Fig. 10. Third mode of structure B

The displacement of each node, reaction and the stresses in the middle of each element of the plate are obtained comparatively with our developed method and the software Sap 2000 as presented in figures 11 and 12. The first two specific modes of vibration of each case are shown in figures 13 and 14. According to these results, a good agreement are obtained.

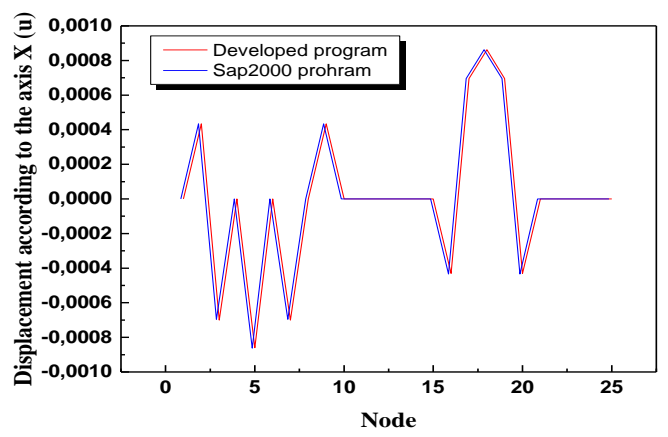

Fig. 11. Nodal displacements in $\mathrm{X}$ direction

According to figure 11, It has been noted that the nodal displacements along the axis ox is maximal in the nodes 25 is zero in the nodes $1,2,3$, 4 and 6 . These results depends on the boundary conditions of the structure.

Table 1. Mesh types

\begin{tabular}{|c|c|c|}
\hline & Developed program & Ansys and Abaqus \\
\hline Elements & 16 & 200 \\
\hline Nodes & 25 & 231 \\
\hline Mesh & Tetrahedral & Tetrahedral \\
\hline
\end{tabular}

Fig. 8. First mode of the structure B 


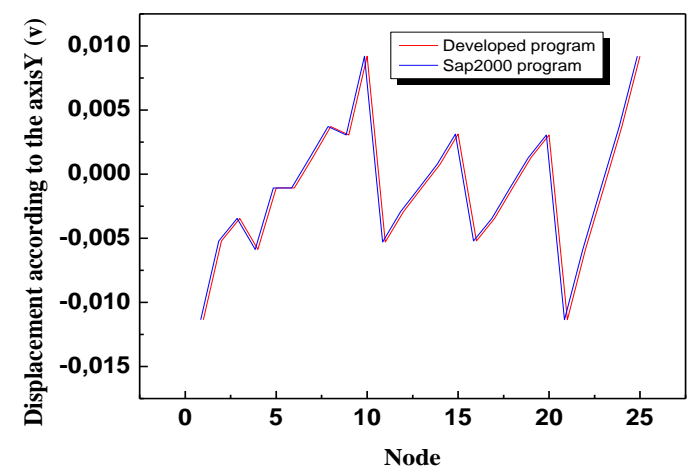

Fig. 12. Nodal displacements in $\mathrm{Y}$ direction

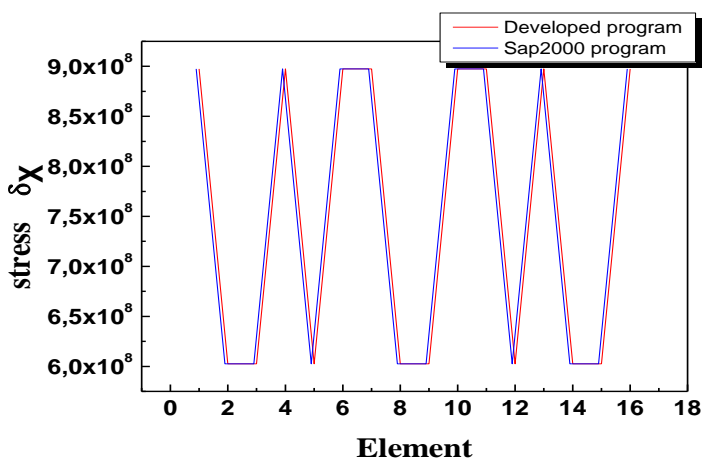

Fig. 13. Stress normal comparison

According to figure 13, it has been noted that the normal stresses for this structure, are symmetrical, which makes it possible to say that this structure with this boundary condition, give us the best results with respect to the normal stresses.

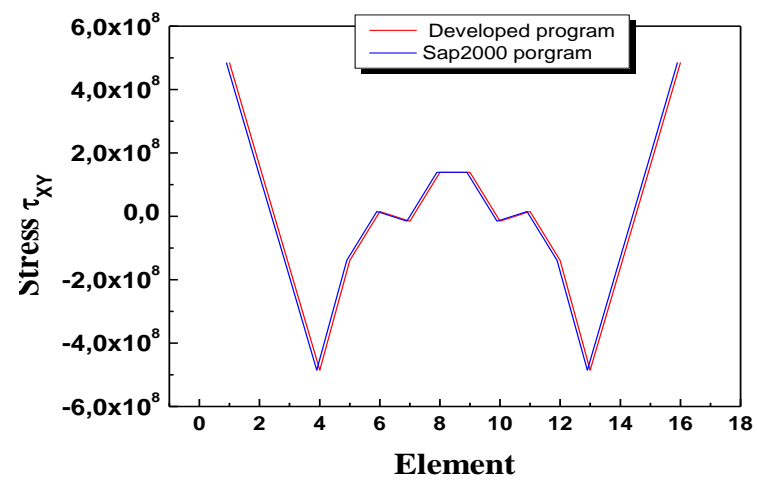

Fig. 14. Comparison of stress tangential

The same thing noticed on the tangential constraints, either on the symmetry of the distribution of these constraints, or on the maximum value and its impact on the structure.

The finite element method is a tool of approximate numerical resolution of the problems of structures and, more generally, physical problems and mechanics and others. It allows the determination of the node displacements of a structure or a plate under any boundary conditions. The essential object of calculation for our case is to determine displacements, the reactions, as well as the stresses in any element of the bi dimensional plate. According to the results found for the various examples that we have considered, we can make the following observations:

The results obtained using our developed program (in the static case):

1-The node displacements in axes $\mathrm{X}$ and $\mathrm{Y}$ direction are very close to the results founds using " Ansys 17.0" and " Abaqus ", software with small for errors 2- For the values of the reactions, it has been noted that the obtained results are completely equivalent to those obtained using "Ansys 17.0" and "Abaqus". The calculation program in a dynamic state gives the eigen frequencies and the eigen values (vibration modes). For the modes, In fact, this study is mainly based on the determination of the eigen modes of the structure. In these condition targeting the first mode of the structure. Therefore, whenever the values of the eigen modes are minimal the structure is in good condition that can withstand vibratory forces, The purpose of this vibratory analysis of a three-dimensional structure (plate element) is the determination of the eigen frequencies of the structure (free vibration).

Different boundary conditions are taking into account. The first three modes of the two structures were determined with different boundary conditions such that the first three modes dominate the vibratory behavior almost 90 percent of the structure. And the fourth and the fifth modes just to see the vibratory response after the three modes. Concerning the nodal displacements it is clear that the structure undergone maximum displacements in the middle which explains the reinforcement of most of the structures, in the two structures. For the axial forces and the reactions at the supports the maximum values concentrate around the most ready elements of the embedding by against they are in the middle of structure in the case of embedding of two sides.

\section{DISCUSSION OF THE RESULTS}

The digital simulation of the mechanical or physical systems is based on several types of methods, in particular finite elements (variationel principles), elements of border (linear equations of field put in the form of integral equations), finite differences (approximations of derived in differential equations or with the derivative partial) and finite volumes (integral form of the conservation equations). The simulation of the mechanical behavior of the deformable solids and the systems made up of such solids, tallies general of this memory, generally rests on the finite element method. This work will be subscribed in to develop a program as a Pascal allowing static and dynamic calculation thin sections in plane state of stress under various boundary conditions, even the determination in static analysis of displacements of each node, reactions, forced in some points of the element and the determination of the frequency and the clean modes in dynamic analysis. The 
comparison between the results obtained and those of software SAP2000 presents a good agreement. The case of the plane stress are be applied to many examples like the structures with thin walls, the structures comprising of the veils of stiffening, the beams out of box and sometimes the hulls (the bridges, the tanks, ships and airplanes).

\section{REFERENCE}

1. Fortin A, Garon A. Les éléments finis de la théorie à la pratique, cours école polytechnique de Montréal, 2011.

https://moodle.polymtl.ca/pluginfile.php/357690/mod resource/content/1/Fortin-Garon-elements-finis.pdf

2. El Ghazi A, El Hajji S. Guide d'utilisation du logiciel MATLAB, département mathématiques et informatique, Université Mohammed V, Maroc 2004.

3. Spiteri P. Présentation générale de la méthode des éléments finis. Ed. Techniques Ingénieur, 2002.

4. Sauer RA, Mergel JC. A geometrically exact finite beam element formulation for thin film adhesion and debonding, Finite Elements in Analysis and Design 2014;86:120-135. https://doi.org/10.1016/i.finel.2014.03.009

5. Alotta G, Failla G, Zingales M. Finite element method for a nonlocal Timoshenko beam model, Finite Elements in Analysis and Design 2014; 89:7792. https://doi.org/10.1016/j.finel.2014.05.011

6. Ghoneim A, A mesh free interface-finite element method for modeling isothermal solutal melting and solidification in binary systems, Finite Elements in Analysis and Design 2015; 95:20-41. https://doi.org/10.1016/j.finel.2014.10.002

7. Kim JG, Lee JK, Yoon HJ. Free vibration analysis for shells of revolution based on p-version mixed finite element formulation, Finite Elements in Analysis and Design 2015; 95:12-19

https://doi.org/10.1016/j.finel.2014.10.006

8. Brighenti R, Bottoli S. A novel finite element formulation for beams with composite cross-section, International Journal of Mechanical Sciences 2014; 89:112-122. ttps://doi.org/10.1016/j.ijmecsci.2014.08.023

9. Donà M, Palmeri A, Lombardo $\mathrm{M}$, Cicirello A. An efficient two-node finite element formulation of multi-damaged beams including shear deformation and rotatory inertia, Computers and Structures 2015;147:96-106. https://doi.org/10.1016/j.compstruc.2014.10.002

10. Teng JG, Fernando D, Yu T. Finite element modelling of debonding failures in steel beams flexurally strengthened with CFRP laminates, Engineering Structures 2015; 86:213-224. https://doi.org/10.1016/j.engstruct.2015.01.003

11. Gao D Y, Machalová J, Netuk H. Mixed finite element solutions to contact problems of nonlinear Gao beam on elastic foundation, Nonlinear Analysis: Real World Applications 2015; 22:537-550. https://doi.org/10.1016/j.nonrwa.2014.09.012

12. Lalanne M, Berthier P, Der Hagobian J. Mécanique des vibrations linéaires, MASSON Edition 1995.

13. Yang T Y, Finite element structural analysis, Prentice-Hall, 1986.

14. Trompette P. Mécanique des structures par la méthode des éléments finis (Statique et dynamique), 1992.

15. Abdi M, Mohamed K. Numerical simulation and active vibration control of piezoelectric smart structure. International Review of Mechanical Engineering 2009; (3)2:175-181.

16. Zienkiewiez OC. The finite element method in engineering analysis, courses, Paris 1991. http://civil.dept.shef.ac.uk/current/module/CIV8130.p df

17. Lebeid A, Necib B. Analysis and numerical modelling of ceramic piezoelectric beam, International Review of Mechanical Engineering 2011; 3:454-456.

18. Brie N. Mise en œuvre d'un calcul de modes propres d'une structure, cours, EDF R\&D 2014.

https://www.code-

aster.org/V2/doc/v11/fr/man_u/u2/u2.06.01.pdf

Received 2017-11-19

Accepted 2018-03-05

Available online 2018-03-12

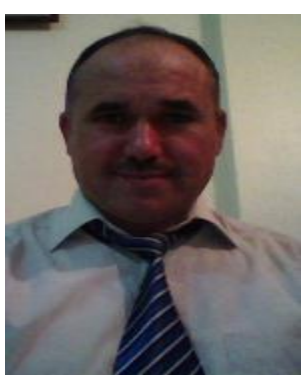

Mr. Fareh HAMRIT was born in M'sila, Algeria, at 07th September 1970. He received his Mechanical Engineering diploma in 1996 and his M.Sc. degrees in Mechanical Engineering in 2006 from Mohamed Boudiaf University of M'sila, Algeria. He has been working for more than ten years with the Department of Mechanical Engineering, University of M'sila, as a Professor. He is interested include in numerical analysis of mechanical problems and in computational solid dynamics (CSD) \& computational structural mechanics. $\mathrm{He}$ is the author or co-author of several technical papers.

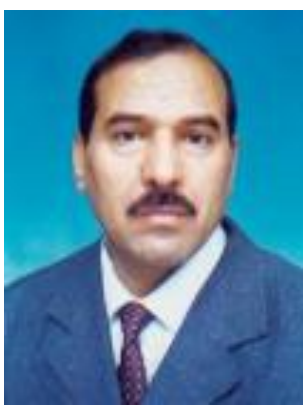

Professor Brahim NECIB born in Constantine Algeria, at 11th February 1953. He received his mechanical engineering diploma in 2001 , his Master degree in mechanical engineering in 1980 from The national polytechnic school, Algiers, Algeria and a $\mathrm{PhD}$ in Aeronautics June 1987, Aeronautical Engineering Department, Purdue University W. Lafayette, Indiana 47906 USA. He is interested in smart Materials, Composite Materials, Complex Structures and Finite Elements, Aerodynamics, Aeroelasticity and Propulsion, Numerical Methods and Modeling (Finite Volume, Finite Elements and Finite Differences), Computational Fluid Dynamics (CFD), Aircraft Stability and Control. He is author and co-author of different articles. $\mathrm{He}$ is the supervisor of many $\mathrm{PhD}$ Students in Algeria. 